\title{
Atypical Chest Pain and Hypertension in a Young Woman
}

\author{
Faisal Ozair
}

\section{ABSTRACT}

Hypertension among young people is fairly common, affecting one quarter of the global population and 1 in 8 adults aged between 20 and 40 years. It is one of the main modifiable risk factors for cardiovascular disease and mortality.

Since many forms of secondary hypertension led to "treatment-resistant" hypertension, it is important to determine the likely causes and this evaluation partly depends on the degree of difficulty in controlling blood pressure.

It is recommended that clinicians should look for the clinical clues that suggest secondary hypertension as it is too expensive and time consuming to perform a complete evaluation for secondary hypertension in every hypertensive patient.

The most common causes of secondary hypertension among young adults are hypothyroidism (1.9\%), renovascular disease $(1.7 \%)$, renal insufficiency $(1.5 \%)$, primary hyperaldosteronism $(1.2 \%)$, Cushing syndrome $(0.5 \%)$, and pheochromocytoma $(<0.3 \%)$.

Keywords: Paraganglioma, Pheochromocytoma, Metanephrines, Young onset Hypertension.

\section{INTRODUCTION}

Presence of hypertension at a young age is associated with a higher risk of cardiovascular events in middle age. It contributes to an earlier onset of coronary heart disease, transient ischemic attacks, stroke \& heart failure. Recognizing secondary causes of hypertension is potentially beneficial, as this might direct specific treatment strategies and potentially cure high blood pressure in young patients.

Chest pain in young adults is not rare. This symptom, however, is frequently atypical in its relationship to exertion and its duration, and after a thorough evaluation, it is often found to be benign in nature - for example, related to chest wall tenderness, costochondritis, hyperventilation, anxiety, mitral valve prolapses or a pulmonary disorder. In other cases, when the chest pain is unrelated to exertion, coronary or oesophageal spasms are other possibilities.

In young patients who present with chest pain, a detailed history should be taken with ischemic cardiac pain in mind before such a symptom is dismissed as benign and noncardiac. If the history is consistent with myocardial ischemia, exercise testing and possibly coronary angiography are indicated to rule out unusual and potentially correctable cardiac abnormalities.
Published Online: January 14, 2021.

ISSN: 2593-8339

DOI: $10.24018 /$ ejmed.2021.3.1.658

Dr Faisal Ozair *

Consultant Endocrinologist \& Diabetes. William Harvey Hospital, East Kent, U.K. (e-mail: faisalozair@yahoo.com)

*Corresponding Author

\section{CASE REPORT}

The following case report describes a young woman with Chest pain \& Hypertension.

30-year-old lady was admitted to the medical assessment unit with severe sudden onset left sided chest pain, radiating to the left arm and jaw which lasted for one hour and was associated with palpitation and sweating. She had history of similar pain for the last one week with minor frequency.

PMH: Hypertension.

Drug history: None.

Social history: Non-smoker \& non-ETOH.

Family History: There is a family history of SDHB gene mutation - father died of metastatic Paraganglioma. Sister has had Paraganglioma removed. She has (according to her sister) never been checked for SHDB mutation.

Highest BP recorded: 165/110. Average BP: 145/100. Pulse 110 / minute. Rest of the examination was normal.

Troponin I: $419 \mathrm{ng} / \mathrm{L}$ and $438 \mathrm{ng} / \mathrm{L}$ (ref 0 -16). Her Renal, Thyroid, Clotting screen including D Dimers were normal.

Was started on Acute Coronary Syndrome protocol \& transferred to CCU, was prescribed Beta blocker but in view of strong family history of Pheochromocytoma, Beta blocker was not dispensed.

CT both adrenals: Adrenal glands appear normal in size and appearance with no focal lesion or nodularity identified. 


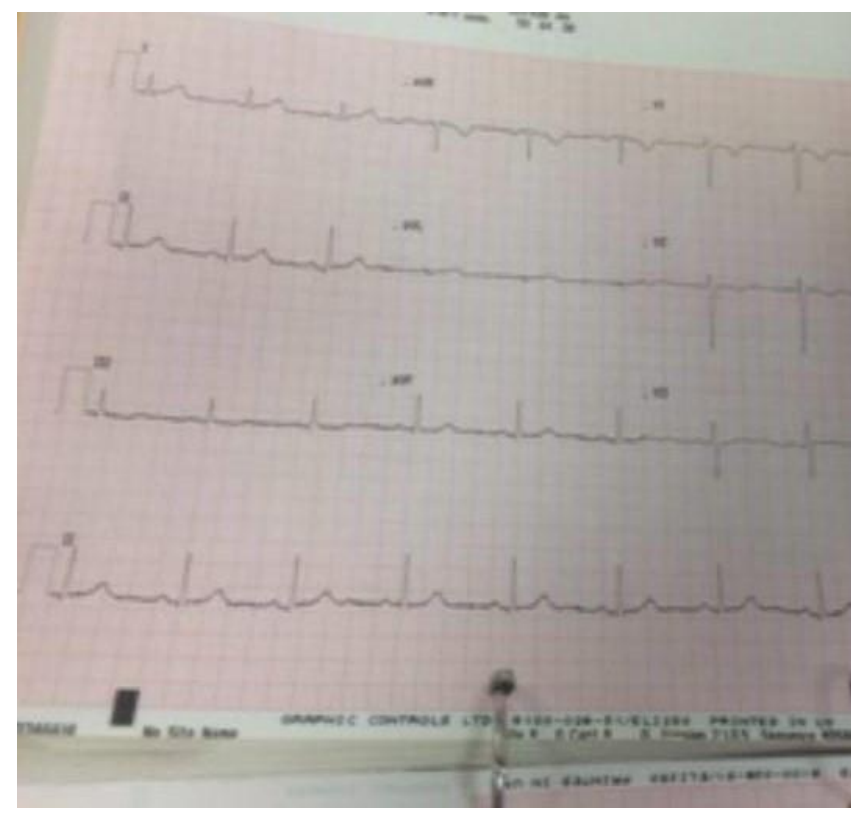

Fig. 1. ECG: $\mathrm{T}$ wave inversion in anterior leads.

Echocardiogram: Severe hypokinesis of mid posterior wall, Normal LV size and wall thickness.

Normal LV systolic function, LVEF 60-70\% visually, Normal RV size and function.

Mild mitral regurgitation.

CT Coronary angiogram: No plaque or stenosis on RCA, LMS \& LAD.

\section{Plasma Metanephrines:}

P Metanephrine (seated): $629 \mathrm{pmol} / \mathrm{L} 0$ - 509.

P Normetanephrine (seated): $\underline{\mathbf{1 5 2 2 7}} \mathrm{pmol} / \mathrm{L} 0$ - 1179.

P 3-Methoxytyramine (seated) $229 \mathrm{pmol} / \mathrm{L}$.

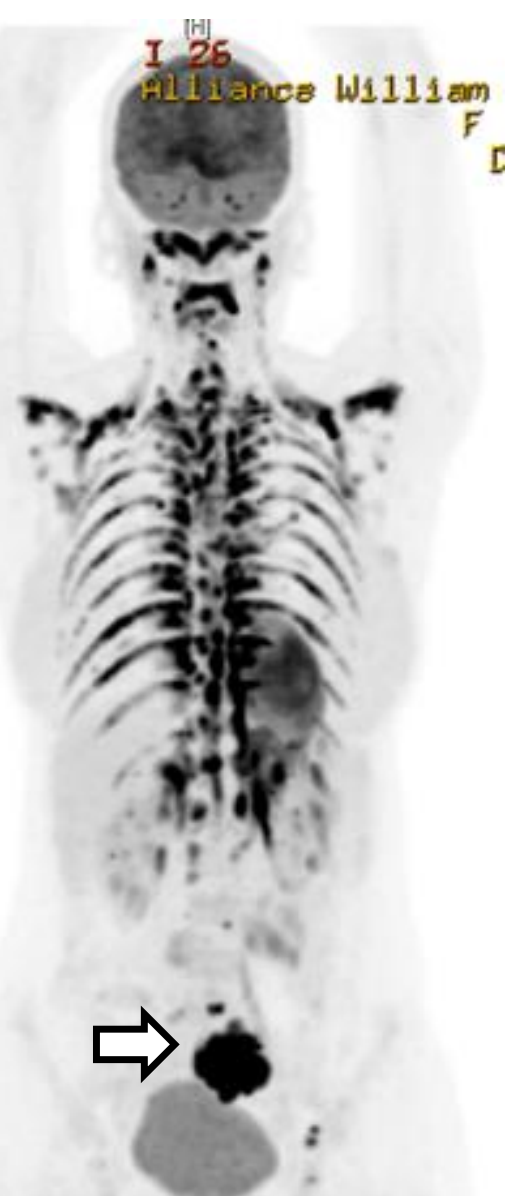

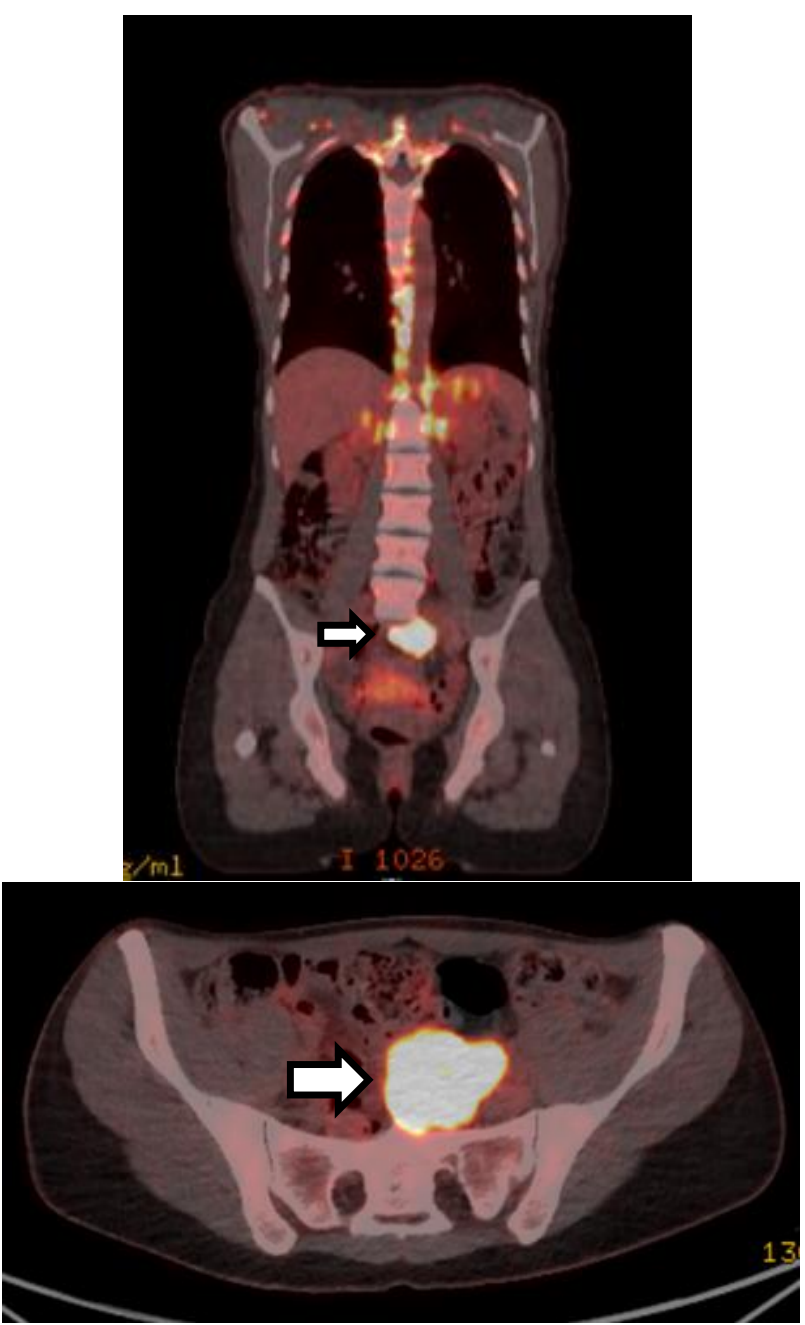
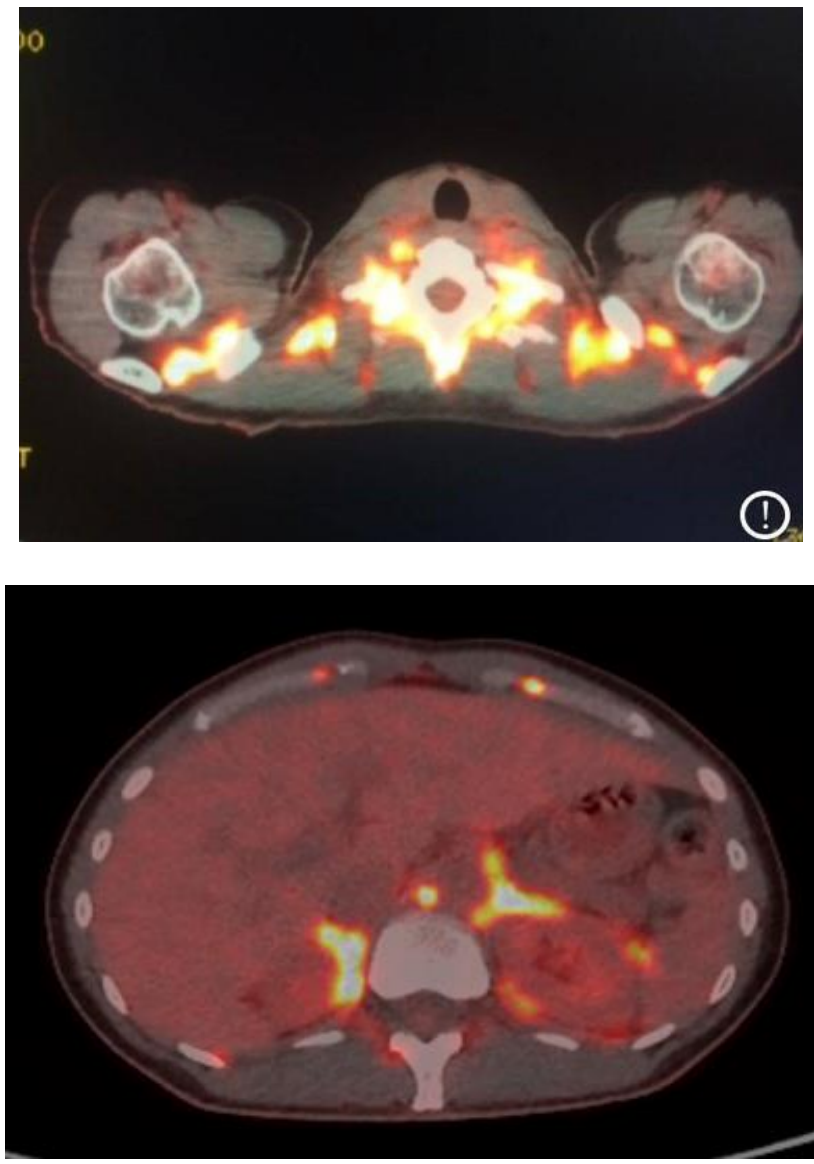

Fig. 2. NM Whole body FDG PET CT. 


\section{NM Whole body FDG PET CT Report}

A malignant Paraganglioma at the level of the pelvic brim with a nodal metastasis at the aortic bifurcation and scattered skeletal metastatic deposits.

\section{Multidisciplinary Meeting Outcome}

The plan is oncological resection of the Paraganglioma (which will remove $>95 \%$ of her visible disease) and then probably adjuvant therapy (with maybe MIBG or Luteteum or Temozolomide or other agents).

Her blood was sent for a gene test for SDHB for confirmation.

Start \& titrate the Doxazosin up to a dose of $2 \mathrm{mg}$ BD if tolerated.

The aim is to achieve long term control over the next 10 years and beyond.

\section{DISCUSSION}

Although Paraganglioma/ Pheochromocytoma is a rare cause of secondary hypertension, but due to strong family history of Paraganglioma in this case, it should be considered as a main differential diagnosis.

Pheochromocytoma crisis is an endocrine emergency associated with higher mortality. Recommended management includes the use of alpha blockade, which is strongly associated with survival of a crisis.

In patients with persistent hypotension, mechanical circulatory supportive therapy is strongly recommended. Surgical intervention is not advisable until patient is medically stabilized.

Paragangliomas are uncommon neuroendocrine tumours that originate from the extra-adrenal autonomic paraganglia, small organs consisting mainly of neuroendocrine cells that are derived from the embryonic neural crest and have the ability to secrete catecholamines.

Catecholamine-secreting paragangliomas often present clinically like pheochromocytomas with episodic headaches, hypertension, tachycardia and sweating. However, the distinction between pheochromocytoma and paraganglioma is important because of implications for associated neoplasms, risk for malignancy, and genetic testing.

Majority of paragangliomas appear to be sporadic. However, approximately one-third to one-half are associated with an inherited syndrome. Some hereditary paragangliomas, have been linked to pathogenic variants in the genes encoding different subunits of the succinate dehydrogenase (SDH) enzyme complex. In addition, susceptibility to pheochromocytomas and paragangliomas is an established component of four genetic syndromes, multiple endocrine neoplasia types $2 \mathrm{~A}$ and $2 \mathrm{~B}$ (MEN2), neurofibromatosis type 1 (NF1), von Hippel Lindau (VHL), and Carney-Stratakis dyad.

$S D H B$-related paragangliomas are usually extra-adrenal and present with an abdominal, pelvic, or thoracic catecholamine-secreting tumor. In general, it is associated with greater morbidity and mortality than other $S D H x$ paraganglioma syndromes. $S D H B$ pathogenic variant carriers develop disease at a relatively young age. $S D H B$ pathogenic variants are also associated with a higher malignancy rate than other types of $S D H x$-associated familial paraganglioma syndromes.

The diagnosis of a secretory paraganglioma can usually be made by measurements of urinary and/or plasma fractionated metanephrines or catecholamines. Biochemical diagnosis should be followed by radiological evaluation (typically either CT or MRI of the abdomen and pelvis) to locate the tumor. If abdominal and pelvic CT or MRI is negative, the next step is cross-sectional imaging of the thorax/head and neck and/or radioisotope (functional) imaging using 18F-fludeoxyglucose (FDG) positron emission tomography (PET) or metaiodobenzylguanidine (MIBG).

Screening for germline pathogenic variants in $S D H$ and other genes should be carried out in all patients with paraganglioma.

\section{LEARNING POINTS}

1) Thorough clinical history, including the family history is crucial in making the diagnosis which is especially vital in this case (i.e., family history of Pheo with SDHB mutation).

2) In the context of Hypertension when secondary cause of Pheochromocytoma is suspected, Beta blocker should be avoided as it precipitates the pheo crisis.

3) Role of Multidisciplinary meeting is very important in managing the complex cases, where different views help to make a unifying management plan.

4) If your clinical suspicion is high, do not be distracted by other features like in this case her chest pain was most likely a cardiac manifestation of Pheo rather than acute coronary syndrome.

5) You need to ask for further investigation, if initial tests results are negative in order to make a diagnosis, e.g., in her case, initial CT adrenal and CT angiogram were negative, but we asked for PET scan which confirmed metastatic Paraganglioma that can easily be missed.

6) Diagnosis of a sporadic paraganglioma or pheochromocytoma should lead to a full genetic workup of the patient and family if SDH mutations are found.

\section{REFERENCES}

[1] Chest Pain in a Young Woman (mayoclinicproceedings.org).

[2] Investigation and Treatment of High Blood Pressure in Young People | Hypertension (ahajournals.org).

[3] Paragangliomas: Epidemiology, clinical presentation, diagnosis, and histology - UpToDate.

[4] Neumann HPH, Young WF Jr, Eng C. Pheochromocytoma and Paraganglioma. N Engl J Med 2019; 381:552.

[5] Evaluation of secondary hypertension - UpToDate.

[6] Calhoun DA, Jones D, Textor S, et al. Resistant hypertension: diagnosis, evaluation, and treatment: a scientific statement from the American Heart Association Professional Education Committee of the Council for High Blood Pressure Research. Circulation 2008; 117:e510. 